\title{
Search for stellar spots in field blue horizontal-branch stars
}

\author{
E. Paunzen ${ }^{1}$, K. Bernhard ${ }^{2,3}$, S. Hümmerich ${ }^{2,3}$, J. Janík ${ }^{1}$, E. A. Semenko ${ }^{4}$, and I. A. Yakunin ${ }^{4}$ \\ 1 Department of Theoretical Physics and Astrophysics, Masaryk University, Kotlářská 2, 61137 Brno, Czech Republic \\ e-mail: epaunzen@physics.muni.cz \\ 2 American Association of Variable Star Observers (AAVSO), 49 Bay State Rd, Cambridge, MA 02138, USA \\ 3 Bundesdeutsche Arbeitsgemeinschaft für Veränderliche Sterne e.V. (BAV), 12169 Berlin, Germany \\ ${ }^{4}$ Special Astrophysical Observatory of the Russian Academy of Sciences, Nizhnii Arkhyz 369167, Russia
}

Received 25 March 2018 / Accepted 5 December 2018

\begin{abstract}
Context. Blue horizontal-branch stars are Population II objects which are burning helium in their core and possess a hydrogen-burning shell and radiative envelope. Because of their low rotational velocities, diffusion has been predicted to work in their atmospheres. In many respects, blue horizontal-branch stars closely resemble the magnetic chemically peculiar stars of the upper main sequence, which show photometric variability caused by abundance spots on their surfaces. These spots are thought to be caused by diffusion and the presence of a stable magnetic field. However, the latter does not seem to be axiomatic.

Aims. We searched for rotationally induced variability in 30 well-established bright field blue horizontal-branch stars in the solar neighbourhood and searched the literature for magnetic fields measurements of our targets.

Methods. We employed archival photometric time series data from the All Sky Automated Survey (ASAS), All-Sky Automated Survey for Supernovae (ASAS-SN), and Wide Angle Search for Planets (SuperWASP) surveys. The data were carefully reduced and processed, and a time series analysis was applied using several different techniques. We also synthesized existing photometric and spectroscopic data of magnetic chemically peculiar stars in order to study possible different surface characteristics producing lower amplitudes.

Results. In the accuracy limit of the employed data, no significant variability signals were found in our sample stars. The resulting upper limits for variability are given.

Conclusions. We conclude that either no stellar surface spots are present in field blue horizontal-branch stars, or their characteristics (contrast, total area, and involved elements) are not sufficient to produce amplitudes larger than a few millimagnitudes in the optical wavelength region. New detailed models taking into account the elemental abundance pattern of blue horizontal-branch stars are needed to synthesize light curves for a comparison with our results.
\end{abstract}

Key words. stars: horizontal-branch - starspots - diffusion - techniques: photometric

\section{Introduction}

Blue horizontal-branch (BHB) stars were first unambiguously detected and defined in Galactic globular clusters (Arp et al. 1952). These stars burn helium in their core and possess a hydrogen-burning shell and a radiative envelope. Owing to their complexity, some aspects of the formation and internal structure of horizontal-branch stars, in general, have been a matter of debate for decades (Catelan 2009). For instance, the blue extension of the horizontal branch varies among clusters, a fact partly associated with metallicity but not entirely explained by it. A number of secondary parameters, such as stellar rotation, cluster concentration, presence of super-oxygen-poor stars, cluster mass, environment of formation, and cluster age have been suggested to provide an explanation for this behaviour, but none of these have been proven completely adequate in describing the complex observational picture (Milone et al. 2014).

The field horizontal-branch (FHB) stars in the solar neighbourhood have been detected in recent decades mainly owing to their kinematic characteristics as they are ideal tracers of the kinematics and dynamics of the thick disk throughout the inner halo of the Milky Way (Wilhelm et al. 1999).

An intriguing phenomenon was first reported by Behr et al. (1999), who found large deviations in element abundances from the expected cluster metallicity for BHB stars in the globular cluster NGC 6205. For example, iron was found to be a factor of three enhanced compared to the solar value, or about 100 times the mean cluster iron abundance. Such atmospheric effects are well known and studied in the classical chemically peculiar (CP) stars of the upper main sequence (Preston 1974), which share the same temperature regime as the BHB stars. Later on, Khalack et al. (2010) found clear evidence of vertical stratification of $\mathrm{Fe}$ in atmospheres of BHB stars. In addition, it was reported that BHB stars with $T_{\text {eff }} \geq 11500 \mathrm{~K}$ have lower rotational velocities than their cooler counterparts (Behr 2003), suggesting that the atmospheres of such stars are stable enough for atomic diffusion to work. The models by Quievy et al. (2009) showed that He sinks in stars with low rotational velocities, which leads to the disappearance of the superficial He convection zone. This then opens the door for atomic diffusion to play a role. The atmospheric models of Hui-Bon-Hoa et al. (2000) showed that the observed photometric jumps and gaps for hot BHB stars can be explained by elemental diffusion in their atmospheres. These models self-consistently calculate the structure of the atmosphere while taking into account the stratification predicted by diffusion (assuming equilibrium). They confirm that vertical stratification of the elements can strongly modify the structure of the atmospheres of BHB stars (LeBlanc et al. 2009). 
Such structural changes of the atmosphere lead to the photometric anomalies discussed above.

For the magnetic $\mathrm{CP}(\mathrm{mCP})$ stars, the overabundant elements in their atmospheres are concentrated into large spot regions that persist for decades to centuries. As an $\mathrm{mCP}$ star rotates, periodic variations in its brightness, spectrum, and magnetic field are observed, in which case the star is referred to as an $\alpha^{2}$ Canum Venaticorum (ACV) variable. These variations led to the development of the oblique rotator model (Stibbs 1950), which explains the periodicity of the variations as a geometrical effect as the star rotates, using a simple dipolar geometry. However, the search for strong magnetic fields in FHB stars was not successful (Elkin 1998).

In this work, we recall the case of the $\mathrm{HgMn}(\mathrm{CP} 3)$ stars, which do not show strong large-scale organized magnetic fields (Kochukhov et al. 2013). However, the line-profile variations detected in the spectra of these stars have also been interpreted in the terms of abundance inhomogeneities (Hubrig et al. 2006). Therefore, rotationally induced photometric variability at some level would be expected. While photometric variations in CP3 stars have been established beyond doubt, the underlying mechanism is still a matter of debate (Morel et al. 2014). However, recent studies strongly favour rotational modulation over pulsation (cf. the discussion in Hümmerich et al. 2018).

Recently, Balona (2017) summarized evidence of the presence of rotational modulation in the Kepler light curves of mid A- to late B-type stars. He concluded that the time-frequency diagrams show stochastic variations in all respects similar to those in spotted cool stars. Following this interpretation, more than half of his sample of stars are proposed to show rotational modulation, indicating that starspots may be the rule rather than the exception among A-type stars. However, these findings still need to be verified with independent photometric time series, and, in particular, spectroscopic data.

Owing to the striking similarities of slowly rotating BHB and classical CP stars, we selected the brightest FHB stars in the solar neighbourhood to search for spot-induced variability. To our knowledge, such an analysis has never been attempted before. Looking at the BHB stars, we also find RR Lyrae variables (For et al. 2011) and low-amplitude g-mode pulsators (Østensen et al. 2012). The latter are technically hot subdwarfs of the sdB type, as they appear as B-type stars with a rather high surface gravity; this places these pulsators below the main sequence in the Hertzsprung-Russell diagram. However, their surface gravities are lower and their helium abundances much higher than in the majority of sdB stars, making them quite unusual.

In total, we present a detailed time series analysis of 30 targets using All Sky Automated Survey (ASAS), All-Sky Automated Survey for Supernovae (ASAS-SN), and Wide Angle Search for Planets (SuperWASP) data. The results and astrophysical characteristics of our sample stars are compared to CP stars.

\section{Target selection, magnetic field measurements, and photometric data treatment}

This section provides information about the employed photometric time series data and describes the processes of target selection, data reduction, and data analysis.

\subsection{Target selection}

We have used the works of Kinman et al. (2000), Behr (2003), and Kafando et al. (2016) as a basis for our investigation. These authors analysed bright FHB stars in the solar neighbourhood and derived astrophysical parameters and elemental abundances. From their list, we excluded RR Lyrae stars because the large amplitude pulsations of these objects interfere with the diffusion mechanism, thereby effectively preventing the formation of stellar spots.

For the remaining stars, we searched for time series in the ASAS, ASAS-SN, and SuperWASP archives. The data from ASAS and SuperWASP were already successfully applied in the analysis of ACV variables (Bernhard et al. 2015a; Hümmerich et al. 2016). In total, 30 stars fulfilled these criteria. Table 1 lists the astrophysical parameters of our target stars. None of these objects have data in the CoRoT (De Medeiros et al. 2013) and Kepler/K2 (Koch et al. 2010; Lund et al. 2016) photometric databases.

\subsection{Magnetic field measurements}

Elkin (1998) presented magnetic field measurements of eight FHB stars, including HD 60778, HD 74721, HD 86986, and HD 161817 from our sample. He used classical Zeeman spectroscopy which was also successfully applied to mCP stars (Bychkov et al. 2009). The magnetic field measurements have been based on circularly polarized spectra obtained with the $6 \mathrm{~m}$ telescope of the Russian Academy of Sciences Special Astrophysical Observatory. The field strength (or corresponding upper limit) was inferred from the relative Zeeman displacements of the spectral lines recorded in the right and left circular polarizations.

For HD 60778, HD 74721, and HD 86986, the following measurements were published: $+15 \pm 115 \mathrm{G},+240 \pm 150 \mathrm{G}$, and $-430 \pm 580 \mathrm{G}$, respectively. For HD 161817, four individual measurements are available: $-550 \pm 140 \mathrm{G},-90 \pm 80 \mathrm{G}$, $+30 \pm 110 \mathrm{G}$, and $+100 \pm 160 \mathrm{G}$. These results seem to indicate the possible presence of a stable magnetic field in HD 161817, which varies over the rotational period. Unfortunately, these are the only measurements available for this star in the literature. Clearly, new spectropolarimetric observations are needed to shed more light on this important issue, although we caution that - as stated above (cf. Sect. 1) - it is still a matter of debate whether a stable magnetic field is a necessary requirement for the formation of stellar spots.

\subsection{Data sources, reduction, and analysis}

The ASAS project aims at continuous photometric monitoring of the whole sky and has the ultimate goal of detecting and investigating any kind of photometric variability. We employed data from the third phase of the project, ASAS-3. The typical exposure time for ASAS-3 $V$-filter observations is three minutes, which results in reasonable photometry for stars in the magnitude range $7<V<14 \mathrm{mag}$. In general, a field is observed one to three times per day (Pigulski 2014).

The ASAS-SN survey has been imaging the entire visible sky every night to a depth of $V<17$ mag (Kochanek et al. 2017). The available data span up to five years of observations. As of end of 2017, ASAS-SN comprises five stations each consisting of four $14 \mathrm{~cm}$ aperture Nikon telephoto lenses. Observations are made using $V$ (two stations) or $g$ (three stations) band filters and three dithered $90 \mathrm{~s}$ exposures. ASAS-SN saturates at 10-11 mag, where the exact limit depends on the camera and the image position. However, a procedure inherited from the ASAS survey is applied which corrects for saturation but introduces a larger noise in the corresponding data sets (Pojmanski 2002). 
E. Paunzen et al.: Search for stellar spots in field blue horizontal-branch stars

Table 1. Astrophysical parameters and their errors, taken from Kinman et al. (2000) and Behr (2003) according to the column "Ref".

\begin{tabular}{|c|c|c|c|c|c|c|c|c|}
\hline Star & TYC & $\begin{array}{c}V \\
(\mathrm{mag})\end{array}$ & $\begin{array}{l}T_{\text {eff }} \\
(\mathrm{K})\end{array}$ & $\log g$ & $\begin{array}{c}{[\mathrm{M} / \mathrm{H}]} \\
(\mathrm{dex})\end{array}$ & $\begin{array}{c}{[\mathrm{Fe} / \mathrm{H}]} \\
(\mathrm{dex})\end{array}$ & $\begin{array}{c}v \sin i \\
\left(\mathrm{~km} \mathrm{~s}^{-1}\right)\end{array}$ & Ref \\
\hline BD-07 230 & $4684-1840-1$ & 11.13 & $9647 \pm 269$ & $3.40 \pm 0.33$ & & $-0.44 \pm 0.11$ & 2 & 2 \\
\hline $\mathrm{BD}+01548$ & $55-357-1$ & 10.76 & $8714 \pm 198$ & $3.38 \pm 0.16$ & & $-2.23 \pm 0.06$ & 10 & 2 \\
\hline $\mathrm{BD}+252602$ & 1994-1778-1 & 10.10 & $8410 \pm 100$ & $3.17 \pm 0.04$ & -2.0 & $-2.08 \pm 0.11$ & 12 & 1 \\
\hline $\mathrm{BD}+422309$ & $3020-678-1$ & 10.84 & $8825 \pm 88$ & $3.20 \pm 0.05$ & -1.5 & $-1.69 \pm 0.05$ & 35 & 1 \\
\hline HD 2857 & $4678-90-1$ & 9.90 & $7566 \pm 58$ & $3.00 \pm 0.08$ & -1.5 & $-1.67 \pm 0.11$ & 30 & 1 \\
\hline HD 4580 & $3659-586-1$ & 8.78 & $8453 \pm 80$ & $3.20 \pm 0.08$ & -1.5 & & 14 & 1 \\
\hline HD 8376 & $2292-1267-1$ & 9.60 & $8133 \pm 48$ & $3.27 \pm 0.04$ & -2.5 & & 10 & 1 \\
\hline HD 13780 & $8045-420-1$ & 9.82 & $7930 \pm 23$ & $3.12 \pm 0.02$ & -1.5 & & 14 & 1 \\
\hline HD 14829 & 5282-1684-1 & 10.30 & $9086 \pm 245$ & $3.31 \pm 0.19$ & & $-2.01 \pm 0.31$ & 14 & 2 \\
\hline HD 31943 & 7593-771-1 & 8.26 & $7893 \pm 59$ & $3.22 \pm 0.11$ & -1.0 & & 7 & 1 \\
\hline HD 60778 & $4830-1230-1$ & 9.09 & $8072 \pm 44$ & $3.13 \pm 0.03$ & -1.5 & $-1.48 \pm 0.06$ & 11 & 1 \\
\hline HD 74721 & $816-2644-1$ & 8.70 & $8908 \pm 88$ & $3.31 \pm 0.02$ & -1.5 & $-1.41 \pm 0.04$ & 2 & 1 \\
\hline HD 78913 & $9195-1020-1$ & 9.28 & $8515 \pm 355$ & $3.25 \pm 0.01$ & -1.5 & & 14 & 1 \\
\hline HD 86986 & 835-1408-1 & 7.98 & $7936 \pm 47$ & $3.19 \pm 0.03$ & -1.5 & $-1.85 \pm 0.05$ & 4 & 1 \\
\hline HD 87047 & $2503-1098-1$ & 9.72 & $7828 \pm 49$ & $3.07 \pm 0.04$ & -2.5 & $-2.36 \pm 0.10$ & 0 & 1 \\
\hline HD 87112 & $3821-809-1$ & 9.70 & $9733 \pm 38$ & $3.48 \pm 0.01$ & -1.5 & $-1.65 \pm 0.07$ & 3 & 1 \\
\hline HD 93329 & $849-785-1$ & 8.77 & $8237 \pm 82$ & $3.12 \pm 0.05$ & -1.5 & $-1.49 \pm 0.08$ & 7 & 1 \\
\hline HD 106304 & $7760-1284-1$ & 9.06 & $9747 \pm 365$ & $3.50 \pm 0.03$ & -1.5 & & 10 & 1 \\
\hline HD 109995 & $3018-494-1$ & 7.59 & $8514 \pm 123$ & $3.08 \pm 0.07$ & -1.5 & $-1.76 \pm 0.09$ & 25 & 1 \\
\hline HD 117880 & $6125-410-1$ & 9.06 & $9285 \pm 131$ & $3.34 \pm 0.09$ & -1.5 & $-2.25 \pm 0.14$ & 13 & 1 \\
\hline HD 128801 & $911-1261-1$ & 8.73 & $10313 \pm 163$ & $3.55 \pm 0.02$ & -1.5 & $-1.38 \pm 0.09$ & 4 & 1 \\
\hline HD 130095 & $6754-156-1$ & 8.14 & $9010 \pm 90$ & $3.30 \pm 0.03$ & -2.0 & & 5 & 1 \\
\hline HD 130201 & $8279-2-1$ & 10.06 & $8645 \pm 275$ & $3.48 \pm 0.03$ & -1.5 & & 16 & 1 \\
\hline HD 139961 & $7849-447-1$ & 8.84 & $8517 \pm 83$ & $3.23 \pm 0.07$ & -1.5 & & 39 & 1 \\
\hline HD 161817 & $2081-3673-1$ & 6.98 & $7533 \pm 10$ & $3.00 \pm 0.01$ & -1.5 & $-1.52 \pm 0.05$ & 17 & 1 \\
\hline HD 167105 & $3536-45-1$ & 8.94 & $9025 \pm 111$ & $3.29 \pm 0.04$ & -1.5 & $-1.62 \pm 0.08$ & 21 & 1 \\
\hline HD 180903 & $6879-187-1$ & 9.57 & $7683 \pm 44$ & $3.10 \pm 0.01$ & -1.5 & $-1.73 \pm 0.08$ & 17 & 1 \\
\hline HD 213468 & $8003-27-1$ & 10.81 & $9147 \pm 68$ & $3.28 \pm 0.02$ & -1.5 & & 12 & 1 \\
\hline HD 252940 & $1885-326-1$ & 9.08 & $7563 \pm 37$ & $2.95 \pm 0.05$ & -1.5 & $-1.70 \pm 0.09$ & 24 & 1 \\
\hline HZ 27 & & 12.81 & $9883 \pm 289$ & $3.38 \pm 0.49$ & & $-1.39 \pm 0.35$ & 7 & 2 \\
\hline
\end{tabular}

Notes. The $[\mathrm{M} / \mathrm{H}]$ values are according to Kinman et al. (2000) whereas $[\mathrm{Fe} / \mathrm{H}]$ is taken from Behr (2003). The $V$ magnitudes are from Kharchenko (2001).

References. 1: Kinman et al. (2000), 2: Behr (2003).

The SuperWASP survey started in 2004 and covers both hemispheres. It provides long-term photometric time series in a broadband filter (4000-7000 $\mathrm{A}$ ) with an accuracy better than $1 \%$ for objects in the magnitude range $8<V<11.5 \mathrm{mag}$ (Pollacco et al. 2006). Observations consist in general of two consecutive $30 \mathrm{~s}$ integrations followed by a 10-minute gap. In this work, we use data from the first and only WASP public data release (Butters et al. 2010) ${ }^{1}$.

Data from the ASAS and SuperWASP surveys have been successfully employed to investigate the small amplitude photometric variability of mCP stars (Bernhard et al. 2015a,b; Hümmerich et al. 2016). These data should therefore be well suited to investigate the possible occurrence of spots in our target stars, which we expect to exhibit similar amplitudes. As yet, ASAS-SN data have not been used for the identification of ACV variables. Before we applied a comprehensive time series analysis, different data reduction steps for the corresponding surveys were performed, which are described in more detail below.

Measurements with quality assignments "C" and "D" were excluded from the ASAS data. The mean $V$ magnitudes were calculated as the weighted average of the values provided in the

\footnotetext{
1 https://wasp.cerit-sc.cz/
}

five different apertures indicated by the ASAS system (Kovács 2005). To check the feasibility of this approach, we subsequently restricted our analysis to the "best" aperture, as indicated by the ASAS system for a given star. No significant differences were found between the two approaches. Finally, a basic $5 \sigma$ clipping was performed to clean the light curves from outliers.

ASAS-SN measurements are taken with different cameras, which we treated separately. The mean for each individual data set was calculated and data points were deleted on a $5 \sigma$ basis. After that, the data of the individual cameras were merged.

We excluded measurements with an error larger than $0.05 \mathrm{mag}$ from the SuperWASP data sets. Each camera was treated separately; the mean for each individual data set was derived, and data points were deleted on a $5 \sigma$ basis. This procedure also corrects for the different offsets of the cameras. As last step, the data of the individual cameras were merged. However, for larger data sets, we also investigated the measurements from each camera separately.

The resulting light curves were examined in more detail using the programme package PeRIOD04 (Lenz \& Breger 2005), which performs a discrete Fourier transformation. The shortest expected periods are about $0.5 \mathrm{~d}$, which was consequently chosen as limit for the investigated period range in the analysis of 
Table 2. Characteristics of the employed time series (time baseline and number of observations) and corresponding upper limits of variability.

\begin{tabular}{|c|c|c|c|c|c|c|c|c|c|}
\hline \multirow[b]{2}{*}{ Star } & \multicolumn{3}{|c|}{ ASAS } & \multicolumn{3}{|c|}{ ASAS-SN } & \multicolumn{3}{|c|}{ SuperWASP } \\
\hline & $\begin{array}{l}\Delta t \\
(\mathrm{~d})\end{array}$ & $N$ & $\begin{array}{c}\mathrm{UL} \\
\text { (mmag) }\end{array}$ & $\begin{array}{l}\Delta t \\
(\mathrm{~d})\end{array}$ & $N$ & $\begin{array}{c}\mathrm{UL} \\
\text { (mmag) }\end{array}$ & $\begin{array}{l}\Delta t \\
(\mathrm{~d})\end{array}$ & $N$ & $\begin{array}{c}\mathrm{UL} \\
(\mathrm{mmag})\end{array}$ \\
\hline BD-07 230 & 3278.1 & 378 & 7.2 & 2189.8 & 970 & 1.7 & & & \\
\hline $\mathrm{BD}+01548$ & 3232.1 & 359 & 5.3 & 1963.6 & 902 & 2.9 & & & \\
\hline $\mathrm{BD}+252602$ & 2267.9 & 206 & 6.2 & 2225.8 & 799 & 1.9 & 1123.1 & 7201 & 2.4 \\
\hline $\mathrm{BD}+422309$ & & & & 2250.7 & 649 & 2.9 & 1476.0 & 5664 & 1.6 \\
\hline HD 2857 & 3296.0 & 410 & 4.5 & 2173.9 & 928 & 3.4 & & & \\
\hline HD 4580 & & & & 1107.9 & 887 & 10.3 & 765.1 & 1425 & 1.4 \\
\hline HD 8376 & & & & 2150.9 & 1284 & 1.2 & 1501.0 & 7511 & 1.0 \\
\hline HD 13780 & 3256.1 & 497 & 4.0 & 1375.6 & 768 & 5.2 & & & \\
\hline HD 14829 & 3296.0 & 469 & 4.7 & 2192.7 & 1007 & 1.8 & & & \\
\hline HD 31943 & 3300.0 & 1013 & 2.8 & 1400.0 & 820 & 11.1 & 151.9 & 557 & 1.4 \\
\hline HD 60778 & 3269.1 & 458 & 3.1 & 2230.9 & 1008 & 3.0 & & & \\
\hline HD 74721 & 2543.1 & 399 & 3.9 & 2217.8 & 651 & 11.8 & & & \\
\hline HD 78913 & 3261.0 & 1523 & 4.0 & 1403.3 & 961 & 4.7 & & & \\
\hline HD 86986 & 2366.7 & 301 & 6.5 & 2155.7 & 667 & 10.3 & & & \\
\hline HD 87112 & & & & 2203.9 & 752 & 3.8 & & & \\
\hline HD 87047 & & & & 2214.0 & 1243 & 1.4 & 777.0 & 3623 & 0.9 \\
\hline HD 93329 & 2864.1 & 403 & 4.2 & 2171.8 & 722 & 6.1 & & & \\
\hline HD 106304 & 3295.0 & 645 & 2.8 & 1391.0 & 815 & 10.0 & 754.2 & 15303 & 1.0 \\
\hline HD 109995 & & & & 2212.0 & 676 & 10.8 & & & \\
\hline HD 117880 & 3198.6 & 657 & 4.1 & 2203.8 & 648 & 13.6 & & & \\
\hline HD 128801 & 2924.1 & 402 & 4.5 & 2218.8 & 749 & 7.8 & & & \\
\hline HD 130095 & 3170.7 & 470 & 4.3 & 2162.7 & 766 & 9.9 & 752.3 & 4192 & 2.3 \\
\hline HD 130201 & 3190.7 & 540 & 3.4 & 731.1 & 565 & 2.9 & 752.3 & 7082 & 1.1 \\
\hline HD 139961 & 3189.6 & 648 & 4.2 & 731.1 & 376 & 18.7 & & & \\
\hline HD 161817 & 2360.6 & 270 & 4.5 & 1996.3 & 859 & 26.3 & & & \\
\hline HD 167105 & & & & 2121.1 & 784 & 10.9 & 1548.0 & 7406 & 0.9 \\
\hline HD 180903 & 3129.7 & 483 & 3.5 & 1452.8 & 727 & 4.2 & 698.3 & 8404 & 1.6 \\
\hline HD 213468 & 3259.1 & 423 & 4.6 & 1333.7 & 774 & 2.8 & 554.7 & 8117 & 1.0 \\
\hline HD 252940 & 2543.1 & 264 & 5.1 & 1179.7 & 435 & 10.4 & 918.8 & 2576 & 1.1 \\
\hline HZ 27 & & & & 2223.8 & 725 & 2.9 & 1476.0 & 9176 & 1.6 \\
\hline
\end{tabular}

all data sets. For rotational velocities larger than that, meridional circulation effectively prevents diffusion in stellar atmospheres (Charbonneau \& Michaud 1991).

The results from PERIOD04 were checked with CLEANEST and phase dispersion minimization (PDM) algorithms as implemented in the programme package PeRAnso (Paunzen $\&$ Vanmunster 2016). The same results were obtained within the derived errors, which depend on the time series characteristics, i.e. the distribution of the measurements over time and photon noise.

Defining the upper limit of variability is not straightforward and has often been discussed in the literature (Reegen et al. 2008). In general, the statistical significance of the noise in the Fourier spectrum is underestimated. We employ a conservative approach and define the upper limit of variability as the upper envelope of the peaks in an amplitude spectrum (Fig. 1). Table 2 gives the characteristics of the employed photometric time series.

\section{Discussion}

The investigated FHB stars share common characteristics with classical CP stars. While these objects are found between spectral types early B to early $\mathrm{F}$, our sample stars cover the $T_{\text {eff }}$ range from about 10500 to $7500 \mathrm{~K}$, which corresponds to spec- tral types B9 to A8 on the main sequence. Thus, neither convection nor stellar winds should significantly affect their atmospheres. In addition, the observed $v \sin i$ values (cf. Table 1) of the FHB stars are well in the range of the values for $\mathrm{CP}$ stars. Therefore, in principle, FHB stars should fulfil the requirements for efficient diffusion in their stellar atmospheres, which is a necessary condition for the formation of atmospheric abundance spots. Furthermore, available magnetic field measurements point to the possible presence of a stable magnetic field in one of our sample stars (HD 161817; cf. Sect. 2.2), although more observations are needed to confirm this. However, we caution that the complete framework and setting in which the " $\mathrm{CP}$ phenomenon" occurs is still not well understood, which also holds true for the prerequisites of efficient diffusion in their stellar atmospheres and the timescales involved (Stift \& Alecian 2016).

For the investigation of the presence of spot-induced variability in our sample of FHB stars, a total of 66 individual data sets with about 125000 individual measurements was analysed. Overlapping data sets from all three surveys exist for eight stars (BD+25 2602, HD 31943, HD 106304, HD 130095, HD 130201, HD 180903, HD 213468, and HD 252940). Time bases range from half a year to almost ten years, which is much longer than the expected rotational periods of up to a few days. With our sample of 30 stars, we can exclude that observational 


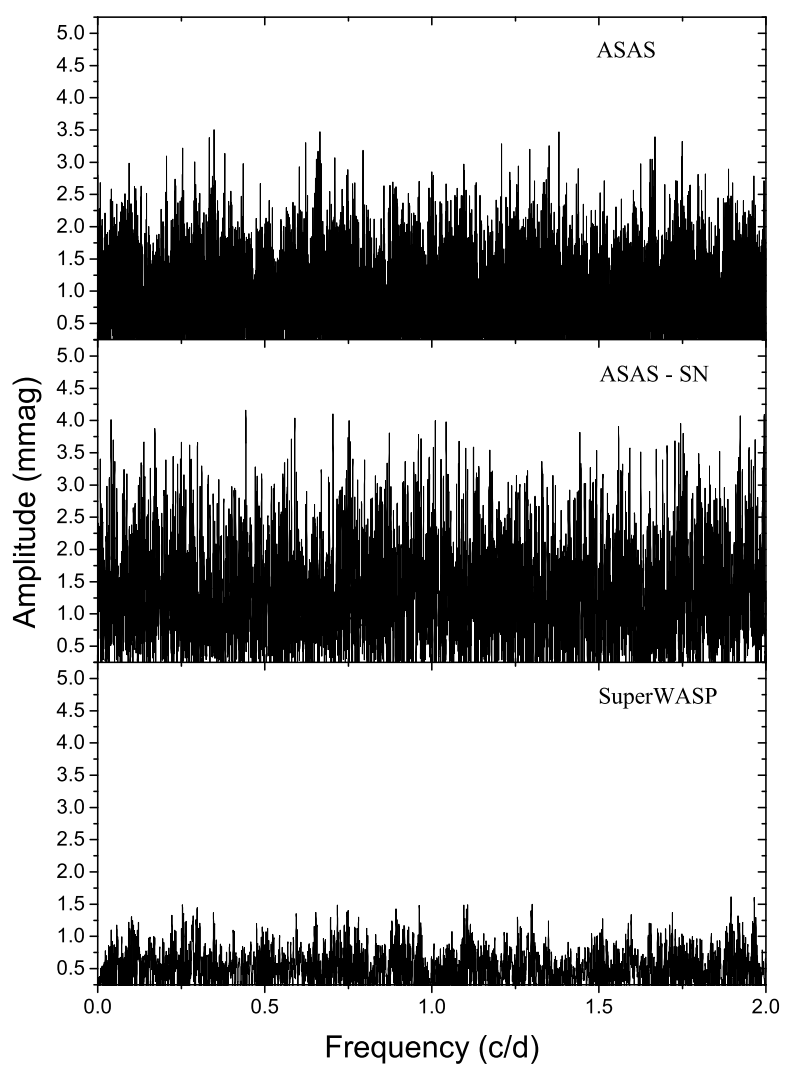

Fig. 1. Amplitude spectra of the ASAS, ASAS-SN, and SuperWASP data for HD 180903. We define the upper limit of variability ("UL" in Table 2) as upper envelope of the peaks in an amplitude spectrum, which is a very conservative definition.

effects such as inclination or rotational periods much longer than the time basis of the photometric data impact our results in a significant way. Thus, given the known $v \sin i$ values and assuming randomly distributed inclination angles (Netopil et al. 2017), rotationally induced variability should be readily detectable with the employed data in this study.

However, we were not able to detect variability with a statistically significant amplitude for any of our sample stars. Table 2 lists the derived upper limits of variability as defined in Sect. 2. As an example, Fig. 1 presents the amplitude spectra for all analysed data sets of HD 180903. Similar results have been obtained for the other targets.

In general, the upper limits derived from SuperWASP data (about $1.5 \mathrm{mmag}$ ) are a factor two lower than the limits derived from ASAS, which is because of the much denser time coverage of SuperWASP. For the ASAS-SN data, we found an obvious correlation between the derived upper limits and the visual magnitudes of the corresponding target stars (Fig. 2). For stars brighter than the ninth magnitude, the upper limits increase up to $25 \mathrm{mmag}$, which is likely due to the treatment of saturated pixels as described in Kochanek et al. (2017). Nevertheless, for the fainter targets, the upper limits derived from ASAS-SN are in line with the values from ASAS and SuperWASP data (Table 2).

ASAS and SuperWASP data have been successfully employed to investigate the spot-induced light changes of ACV variables (Bernhard et al. 2015a,b; Hümmerich et al. 2016). We compared the derived upper limits for our FHB stars to the results for ACV variables published by these investigators. As the ACV stars are also within the same magnitude range as our

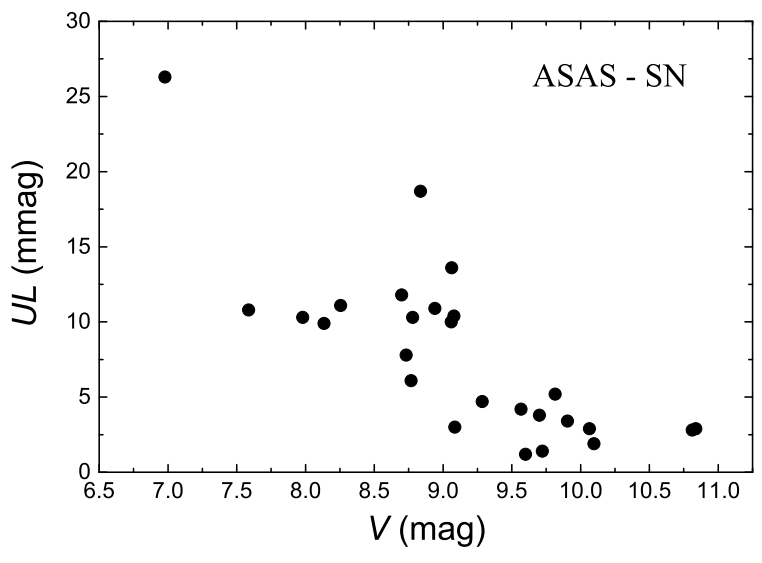

Fig. 2. Dependence of the upper limit for variability (UL) on the visual magnitude of the target stars from ASAS-SN data. Owing to nonlinearity effects, the values for stars brighter than 9th magnitude are much larger than for the fainter targets.

sample stars, the quality of the employed time series data is comparable, which renders a direct comparison possible. However, it is important to note that the ACV samples are significantly dominated by $\mathrm{A} 0 \mathrm{Si}$ stars $\left(T_{\mathrm{eff}}\right.$ of about $\left.10000 \mathrm{~K}\right)$, which are known to exhibit the largest photometric amplitudes among $\mathrm{mCP}$ stars.

ACV variables exhibit variability amplitudes from 3 to $35 \mathrm{mmag}$ in ASAS data (mean value of $10 \mathrm{mmag}$ ) and $2.8-53 \mathrm{mmag}$ (mean value of $11 \mathrm{mmag}$ ) in SuperWASP data. Unfortunately, no information about the non-variable mCP stars and the corresponding upper limits are given in the above-listed references. However, the lowest detected amplitudes are about $2 \mathrm{mmag}$ and the noise should be at least a factor of two less (Hümmerich et al. 2016). Therefore, typical ACV-like variations would have been readily detectable in most of the data sets we employed for the analysis of our sample FHB stars, which suggests that either no spots are present or the resulting photometric amplitudes are too small for detection in the employed data.

To tackle this question, we investigated possible sets of spot parameters that satisfy the observations, i.e. those resulting in very low-amplitude variability. It is important to note that from the derived upper limits, it is not possible to derive limits for spot sizes straightforwardly. The spot-induced amplitude for a given wavelength range (or filter) depends not only on the size, contrast, and distribution of the spots, but also on the involved elements (Krtička et al. 2013; Prvák et al. 2015). Furthermore, the abundance patterns of BHB stars are very much different from CP stars (Kinman et al. 2000) and no specialized models exist to synthesize light curves for a given surface distribution of stellar spots in these objects.

As a near approximate, we therefore had to resort to the available information for several well-described mCP stars covering the $T_{\text {eff }}$ range of our targets: HD 3980 (Nesvacil et al. $2012, T_{\text {eff }}=8300 \mathrm{~K}$ ), HD 112185 (Lüftinger et al. 2003, $T_{\text {eff }}=$ $9000 \mathrm{~K}$ ), and HD 124224 (Krtička et al. 2012, $T_{\text {eff }}=13000 \mathrm{~K}$ ). Synthetic light curves in the filters employed by the ASAS ( $V$ filter), ASAS-SN ( $V$ and $g$ filters), and SuperWASP (broadband filter, 4000-7000 ̊) surveys were calculated using the available surface abundance maps, TLUSTY model atmospheres, and the SYNSPEC code.

Light curves were calculated assuming spots of different sizes and contrast. If the spot contrast is kept fixed, a $50 \%$ reduction of the spot area reduces the photometric amplitude 
by a factor of four in all models. On the other hand, if the area covered by spots is kept constant, a contrast reduction of $80 \%$ is needed to achieve an amplitude change of the same magnitude. Assuming different amplitudes and keeping the spot contrast fixed, we find a clear correlation between the spot area and the resulting photometric amplitude, in the sense that spot size decreases linearly with light curve amplitude. Thus, if spots are present in our sample stars, either their contrast is much lower or, much more likely, the total area covered by spots is much smaller. This suggests that the surface characteristics of BHB and $\mathrm{mCP}$ stars are very different. However, this finding has been based on small number statistics and needs to be confirmed by the investigation of a larger sample of stars.

In summary, we conclude that either no stellar surface spots are present in our sample stars, or the resulting photometric amplitudes are very small and below the detection limit of our photometric data.

\section{Conclusions and outlook}

We have searched for rotationally induced variability within 30 well-established bright FHB stars. We were not able detect statistically significant photometric variability in our target stars. Either no stellar spots are present or their characteristics (contrast, total area, and involved elements) are not sufficient to produce amplitudes larger than a few millimagnitudes in the optical wavelength region. Up to now, no corresponding models exist that reliably predict photometric amplitudes for the variety of surface configurations and abundance patterns observed in BHB (and $\mathrm{mCP}$ ) stars.

The next logical step is to search for corresponding variations in highly accurate space-based data. Unfortunately, no data for our targets are present in the CoRoT and Kepler databases. Another important step will be the collection of highly accurate spectropolarimetric measurements of all bright FHB stars in the solar neighbourhood to search for the presence of stable magnetic fields, which will also be important for the modelling of synthetic light curves. In consequence, the combination of observational and theoretical efforts will shed more light on whether diffusion on the blue horizontal branch is able to produce spotted surfaces as has been shown for main sequence stars.

Acknowledgements. This project was supported by the grants 7AMB17AT030 (MŠMT), 16-01116S (GA ČR), and No. 14-50-00043 (Russian Science Foundation). This paper makes use of data from the DR1 of the WASP data (Butters et al. 2010) as provided by the WASP consortium, and the computing and storage facilities at the CERIT Scientific Cloud, reg. no. CZ.1.05/3.2.00/08.0144, which is operated by Masaryk University, Czech Republic.

\section{References}

Arp, H. C., Baum, W. A., \& Sandage, A. R. 1952, AJ, 57, 4

Balona, L. A. 2017, MNRAS, 467, 1830

Behr, B. B. 2003, ApJS, 149, 101

Behr, B. B., Cohen, J. G., McCarthy, J. K., \& Djorgovski, S. G. 1999, ApJ, 517, L135

Bernhard, K., Hümmerich, S., Otero, S., \& Paunzen, E. 2015a, A\&A, 581, A138 Bernhard, K., Hümmerich, S., \& Paunzen, E. 2015b, Astron. Nachr., 336, 981 Butters, O. W., West, R. G., Anderson, D. R., et al. 2010, A\&A, 520, L10 Bychkov, V. D., Bychkova, L. V., \& Madej, J. 2009, MNRAS, 394, 1338 Catelan, M. 2009, Ap\&SS, 320, 261

Charbonneau, P., \& Michaud, G. 1991, ApJ, 370, 693

De Medeiros, J. R., Ferreira Lopes, C. E., Leão, I. C., et al. 2013, A\&A, 555, A63

Elkin, V. G. 1998, Contrib. Astron. Obs. Skalnate Pleso, 27, 452

For, B.-Q., Sneden, C., \& Preston, G. W. 2011, ApJS, 197, 29

Hubrig, S., González, J. F., Savanov, I., et al. 2006, MNRAS, 371, 1953

Hui-Bon-Hoa, A., LeBlanc, F., \& Hauschildt, P. H. 2000, ApJ, 535, L43

Hümmerich, S., Paunzen, E., \& Bernhard, K. 2016, AJ, 152, 104

Hümmerich, S., Niemczura, E., Walczak, P., et al. 2018, MNRAS, 474, 2467

Kafando, I., LeBlanc, F., \& Robert, C. 2016, MNRAS, 459, 871

Kharchenko, N. V. 2001, KFNT, 17, 409

Khalack, V., LeBlanc, F., \& Behr, B. B. 2010, MNRAS, 407, 1767

Kinman, T., Castelli, F., Cacciari, C., Bragaglia, A., Harmer, D., \& Valdes, F. 2000, A\&A, 364, 102

Koch, D. G., Borucki, W. J., Basri, G., et al. 2010, ApJ, 713, L79

Kochanek, C. S., Shappee, B. J., Stanek, K. Z., et al. 2017, PASP, 129, 104502

Kochukhov, O., Makaganiuk, V., Piskunov, N., et al. 2013, A\&A, 554, A61

Kovács, G. 2005, A\&A, 438, 27

Krtička, J., Mikulášek, Z., Lüftinger, T., et al. 2012, A\&A, 537, A14

Krtička, J., Janík, J., Marková, H., et al. 2013, A\&A, 556, A18

Lanz, T., \& Hubeny, I. 2003, ApJS, 146, 417

Lanz, T., \& Hubeny, I. 2007, ApJS, 169, 83

LeBlanc, F., Monin, D., Hui-Bon-Hoa, A., \& Hauschildt, P. H. 2009, A\&A, 495, 937

Lenz, P., \& Breger, M. 2005, Commun. Asteroseismol., 146, 53

Lüftinger, T., Kuschnig, R., Piskunov, N. E., \& Weiss, W. W. 2003, A\&A, 406, 1033

Lund, M. N., Chaplin, W. J., Casagrande, L., et al. 2016, PASP, 128, 124204

Mathur, S., Huber, D., Batalha, N. M., et al. 2017, ApJS, 229, 30

Milone, A. P., Marino, A. F., Dotter, A., et al. 2014, ApJ, 785, 21

Morel, T., Briquet, M., Auvergne, M., et al. 2014, A\&A, 561, A35

Nesvacil, N., Lüftinger, T., Shulyak, D., et al. 2012, A\&A, 37, A151

Netopil, M., Paunzen, E., Hümmerich, S., \& Bernhard, K. 2017, MNRAS, 468, 2745

Østensen, R. H., Degroote, P., Telting, J. H., et al. 2012, ApJ, 753, L170

Paunzen, E., \& Vanmunster, T. 2016, Astron. Nachr., 337, 239

Pigulski, A. 2014, Precis. Asteroseismol., 301, 31

Pojmanski, G. 2002, Acta Astron., 52, 397

Pollacco, D. L., Skillen, I., Collier Cameron, A., et al. 2006, PASP, 118, 1407 Preston, G. W. 1974, ARA\&A, 12, 257

Prvák, M., Liška, J., Krtička, J., Mikulášek, Z., \& Lüftinger, T. 2015, A\&A, 584, A17

Reegen, P., Gruberbauer, M., Schneider, L., \& Weiss, W. W. 2008, A\&A, 484, 601

Quievy, D., Charbonneau, P., Michaud, G., \& Richer, J. 2009, A\&A, 500, 1163

Stibbs, D. W. N. 1950, MNRAS, 110, 395

Stift, M. J., \& Alecian, G. 2016, MNRAS, 457, 74

Wilhelm, R., Beers, T. C., Sommer-Larsen, J., et al. 1999, AJ, 117, 2308 